Methods Fifty-six patients with GI symptoms > 6 months after radical pelvic radiotherapy underwent structured gastroenterological assessment as part of a service evaluation. They were assessed using the following questionnaires: inflammatory bowel disease questionnaire(IBDQ); Vaizey incontinence questionnaire (VIO); and the Common Terminology Criteria for Adverse Events (CTCAE) pelvic symptom questionnaire.12 month assessments were compared to the previously reported baseline and 6 month assessments to determine if the improvement in symptoms was sustained. Patient satisfaction with the service was assessed at 12 months by an in-house questionnaire.

Results Forty patients(71\%)completed the 12 month assessment and $37(66 \%)$ completed the patient satisfaction questionnaire. The initial statistically significant improvement in GI symptoms from baseline to 6 months in parallel to GI evaluation was sustained up to 12 months in all questionnaires (IBDQ $p=0.019$, IBDOB and CTCAE rectum bowel subset $p<0.0005)$ except the VIQ ( $p=0.098)$. There was also a clinically significant improvement as defined by an increase in IBDQ score of $\geq 0.5$ points per question. Median total $\mathrm{IBDQ}$ and $\mathrm{IBDOB}$ score increased by 25 and 11 points respectively between baseline and 12 months. $97 \%$ of patients found the appointments convenient, $97 \%$ felt their problems were understood; $86 \%$ were satisfied with the outcome and $89 \%$ with the service. Dissatisfaction related to communication $(\mathrm{n}=3)$, travel $(\mathrm{n}=2)$ and ongoing symptoms $(\mathrm{n}=3)$.

Conclusion The clinically and statistically significant improvement in GI symptoms found in parallel to structured gastroenterological evaluation for chronic GI symptoms following pelvic radiotherapy was sustained over 12 months follow up. These data suggest that structured investigation on the basis of the BSG guidelines can lead to a sustained improvement in symptoms and is acceptable to patients. Further research is essential to optimise patient care.

Disclosure of Interest None Declared.

\section{PTU-187 IS PLASMA CITRULLINE CONCENTRATION A RELIABLE MARKER FOR DIAGNOSIS AND CLINICAL MANAGEMENT OF COELIAC DISEASE ?}

doi:10.1136/gutjnl-2013-304907.277

$1,2,{ }^{*} \mathrm{C}$ Papadia, ${ }^{2} \mathrm{~F}$ Fonaroli, ${ }^{3} \mathrm{~F}$ Lanzarotto, ${ }^{4} \mathrm{M}$ Salemme, ${ }^{5} \mathrm{~A}$ Di Sabatino, ${ }^{2} \mathrm{G}$ de'Angelis, ${ }^{4} \mathrm{~V}$ Villanacci, ${ }^{5} \mathrm{G}$ R Corazza, ${ }^{1} \mathrm{~A}$ Forbes. ${ }^{1}$ Gastroenterology \& Nutrition, University College London, London, UK; ${ }^{2}$ Gastroenterology, Parma University Hospital, Parma; ${ }^{3}$ Gastroenterology; ${ }^{4}$ Pathology, Spedali Civili Brescia, Brescia; ${ }^{5}$ Internal Medicine, IRCS San Matteo, Pavia, Italy

Introduction In chronic villous atrophy plasma citrulline concentration (PCC) is decreased at the same severity and extent of mucosal lesions of villous architecture. Marsh-Oberhuber classification is conventionally used for grading villous atrophy in coeliac disease and a correlation with plasma citrulline concentrations has been found in pioneering studies. The Corazza-Villanacci classification gives better inter-observer agreement then Marsh-Oberhuber classification. Our primary aim was to correlate PCC to Corazza-Villanacci classification in coeliac disease. We aimed also to yield information in respect of PCC after one year of gluten free diet.

Methods Forty subjects with a diagnosis of acute celiac disease have been studied. Nine out of forty patients were on gluten challenge diet. All patients underwent OGD with multiple biopsies and a blood test for plasma citrulline concentration at baseline and after one year of gluten free diet (GFD). Routine haematological and biochemical investigations were performed including, IgA tTG, IgA EMA and IgA/G antigliadin, ESR, haemoglobin and haematinics, albumin, liver function tests and creatinine. BMI and clinical symptoms were monitored. Histology was interpreted according to Marsh-Oberhuber and Corazza-Villanacci Classification. Plasma citrulline concentration was analysed by High Performance Liquid Chromatography
Results Mean plasma citrulline concentration was lower $(15.12 \mu \mathrm{mol} / \mathrm{l})$ at baseline, in patients with active celiac disease, than in the same group of patients after one year of GFD $(16.47 \mu \mathrm{mol} / \mathrm{l})$ however we did not observe any overall change in citrulline concentration after one year of gluten-free diet. All patients were only partially histopathologically and clinically responsive to one year of GFD. Plasma citrulline concentrations correlated with VillanacciCorazza classification $(\mathrm{P}=0.05)$ in patients on gluten challenge diet. Patients with a score of 2 had lower citrulline values compared to those with a score of 1 , on average 4 units. Correlation was not found between plasma citrulline concentrations and Marsh-Oberhuber classification at baseline and after one year of gluten-free diet.

Conclusion Plasma citrulline concentration may be considered a reliable marker of severity and extent of small bowel villous atrophy in acute coeliac disease, more data are warranted to determine its role in the long-term management.

Disclosure of Interest None Declared.

\section{PTU-188 REPEAT VIDEO CAPSULE ENDOSCOPY- IS IT WORTH IT?}

doi:10.1136/gutjnl-2013-304907.278

1.*C Parker, 'P Rajasekhar, 'R Bevan, ' $\mathrm{C}$ Davison, 'S Panter. 'Gastroenterology, South Tyneside District Hospital, South Shields, UK

Introduction Few studies have reported on the yield of repeat capsule endoscopy (CE) in the same patient; data regarding this diagnostic strategy are limited. ${ }^{1,2}$ The aims of this work were to assess the indications for repeat capsule and to determine the diagnostic yield of repeat capsule in our trust.

Methods A retrospective review of all patients who underwent CE at South Tyneside District Hospital between August 2004 and October 2012 was conducted. Patients who underwent a repeat CE were identified and divided into one of four subgroups. Findings were classified as positive or negative; positive findings were taken as presence on report of ulcers, tumours, strictures, polyps, blood or angioectasia.

Results A total of 1083 studies were performed, 83 were repeat studies. 7 patients were noted to have greater than 2 repeats.

\section{Indications}

Group 1 Gastric retention or technical failure $(\mathrm{N}=16)$

Group 2 Surveillance $(\mathrm{N}=7)$

Group 3 Poor views (as commented on by reporting physician on report) or incomplete (not seen to enter the colon) on initial study $(\mathrm{N}=31)$

Group 4 Ongoing symptoms/assessment of disease extent/ unclear findings on initial VCE $(\mathrm{N}=36)$ ( 7 cases are reported in both group 3 and 4)

Yield Overall yield, excluding gastric retention was 38\% for the first study and $46 \%$ for $2^{\text {nd }}$ study, of those with an initial negative study (42 patients), $21 \%$ of these had a positive repeat. (those with poor views had been given bowel preparation, those with an incomplete capsule study had a capsule recording time of 8-9 hours on both studies).

Positive findings

\section{Abstract PTU-188 Table}

\begin{tabular}{lll}
\hline Group & Positive findings $\mathbf{1}^{\text {st }}$ study & Positive findings $\mathbf{2}^{\text {nd }}$ study \\
\hline 1 & $\mathrm{~N} / \mathrm{A}$ & $5 / 16(31 \%)$ \\
2 & $4 / 7(57 \%)$ & $4 / 7(57 \%)$ \\
3 & $3 / 31(10 \%)$ & $10 / 31(32 \%)$ \\
4 & $16 / 36(44 \%)$ & $17 / 36(47 \%)$ \\
\hline
\end{tabular}

Subgroup analysis group 4:

- Ongoing symptoms with consistent with ?Crohn's or known Crohn's the yield remains the same on $1^{\text {st }}$ and $2^{\text {nd }}$ capsule $4 / 9(44 \%)$. 